“C 2019/2020 IEEE. Personal use of this material is permitted. Permission from IEEE must be obtained for all other uses, in any current or future media, including reprinting/republishing this material for advertising or promotional purposes, creating new collective works, for resale or redistribution to servers or lists, or reuse of any copyrighted component of this work in other works." 


\title{
Neutral-point Voltage Control of Three-level NPC Inverter for Three-phase APF based on Zero-sequence voltage injection
}

\author{
Muhammad Kashif*, M.J. Hossain, Vivek Sharma, S.M. Nawazish Ali, Asif Khan
}

\begin{abstract}
Active Power Filter (APF) has already adopted the three-level inverter topology in medium voltage and highpower application for solving power quality problems. Neutralpoint voltage clamped (NPC) Inverter has become matured and widely used topology due to its robustness. However, neutralpoint voltage at the $\mathrm{DC}$-side need to be maintained as close to zero as possible. This paper focusses on the neutral point voltage control of the three-level NPC inverter based on multicarrier PWM by manipulating dwell time of small vectors by injecting zero-sequence voltage in modulating signal. The effectiveness of the presented method on three-level NPC inverter is validated via simulation in MATLAB/ Simulink. The results confirms the potentiality of the method in maintaining the neutral point voltage to minimum value with overall desired good APF compensation characteristics.
\end{abstract}

\section{INTRODUCTION}

Industrial loads are mostly non-linear loads such as power electronic converters, frequency changers and motor drives are the prime sources for the harmonic pollution in power system. These non-linear devices not only deteriorate the power quality and power factor but also result in equipment failure connected to the grid [1], [2]. The Active Power Filters (APF) are the most viable solution to improve the power quality by mitigating these harmonics from power system [3], [4]. Although, there is a variety of two-level APF available for low voltage (below 690 volts) applications yet, for medium and high voltage filtering, APF with multilevel converter topologies is an optimal solution [5]. Unlike twolevel voltage source converters, multilevel converters consist of cascaded sub-modules which improve waveform quality thereby reducing the losses and electromagnetic interference. Thus, multilevel converters such as (NPC) specifically has gained more attention in the research community in recent years [6], [7].

Although NPC is very popular for its performance, however, it has voltage unbalancing issue at its neutral point. This issue is more considerable when operating conditions such as load variations and modulation index are changed.
This further enhances the voltage difference among capacitors; which eventually shifts the neutral point of NPC. The resulting imbalances do not only cause damages to switching devices, but it also introduce lower order harmonics in output voltage. As a result, the overall THD of the output current is increased [9]. There are several reasons for the change in dc link voltages which are:

- Inaccuracies during manufacturing process.

- Imbalance operation in three phases due to variations in operating conditions.

- Unreliable switching device characteristics.

- The differences caused by small voltage vector dwell time may influence the potential neutral point deviation.

As a rule of thumb, the voltage across each dc link capacitor in three level NPC must be half of the overall dc link voltage that can only be achieved via switching control algorithms. In literature, several neutral point voltages balancing schemes for space vector and carrier-based modulation techniques have been presented [9]-[11].

This paper focuses on sinusoidal PWM based zerosequence voltage injection algorithm for neutral point voltage balancing in three-level NPC inverter for shunt APF.The algorithm is then verified and implemented in NPC inverter based APF in MATLAB/Simulink which shows the effectiveness of the algorithm.

\section{Three-LeVel SAPF Control Structure}

A generalized control architecture for the three phase SAPF is shown in Fig. 1. The control system comprises of four sub-systems that includes reference current estimation, DC side voltage control, inner current controller and a feedforward neutral point voltage controller. APF based threelevel NPC inverter is the same as two-level inverter except a feed forward control is used to control neutral point voltage. In order to achieve compensation characteristics, the DClink voltage should be at least as high as the nominal line to 
netural voltage, given as

$$
v s \leq V d c \leq 2 v s
$$

Compared to two-level inverter, the DC-side capacitor voltage rating in three-level NPC inverter is half of the total dc side voltage, and the minimum capacitance can be found by [11]

$$
C \geq \frac{\left|\int_{0}^{t} i d t\right|}{4 \Delta V_{\max }}
$$

" $i$ " represents injecting current, and $\Delta V_{\max }$ " is the maximum allowable ripple voltage on DC-side capacitors.

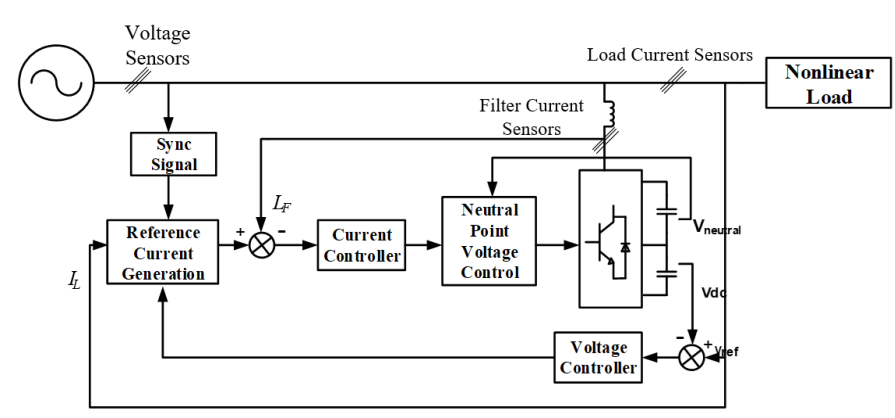

Fig. 1: Generalized Block of Three-level Shunt APF

\section{TOpology Review AND MUlti-CARRIER PWM}

The NPC inverter comprises of the bidirectional switching devices (e.g. IGBTs, MOSFETs etc) and clamping diodes. The dc-side of NPC inverter consists of two capacitors which divide the dc-link voltage into three different voltage levels as shown in Fig. 2. The pair of switches operate in complementary fashion such that when switches $S_{1}$ and $S_{3}$ are ON, $S_{2}$ and $S_{4}$ are OFF and vice versa. The converter output is connected to $V_{d c} / 2$ when $S_{1}$ and $S_{2}$ are $\mathrm{ON}$; whereas, the output is $-V_{d c} / 2$ when $S_{3}$ and $S_{4}$ are ON.

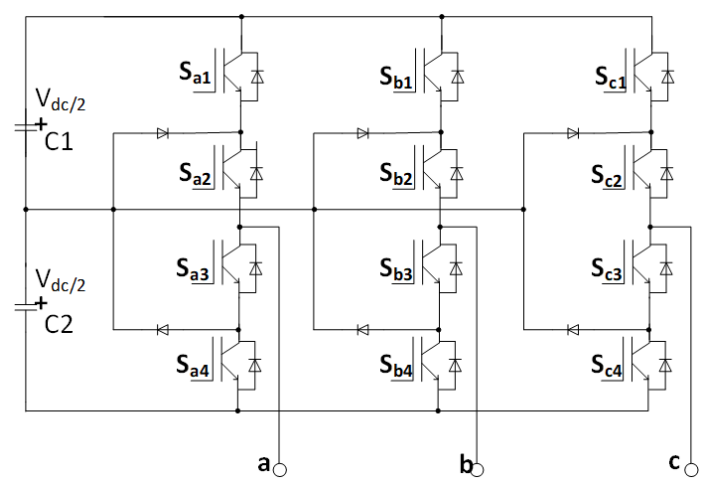

Fig. 2: Three-level NPC Inverter Topology
The different switching states for the NPC has been provided in Fig. 3 There are 27 (i.e. $3^{3}$ ) different switching states in total for the three level NPC that are further bifurcated into small, medium, and large vectors. It is worth noting that not all vectors influence the neutral point potential. Since zero and large vectors do not connect any phase to neutral point, they do not influence neutral-point voltage. However, small and medium vectors do affect the voltage by connecting one or two phases to neutral-point and neutrapoint voltage may rise or drop depending upon the current direction of the neutral point potential. Three phase positive sequence modulation voltage can be expressed,

$$
\left\{\begin{array}{l}
e_{a}=m \cos (\omega t) \\
e_{b}=m \cos \left(\omega t-\frac{2 \pi}{3}\right) \\
e_{c}=m \cos \left(\omega t+\frac{2 \pi}{3}\right)
\end{array}\right.
$$

where $m$ is the modulation index. Sinusoidal PWM is

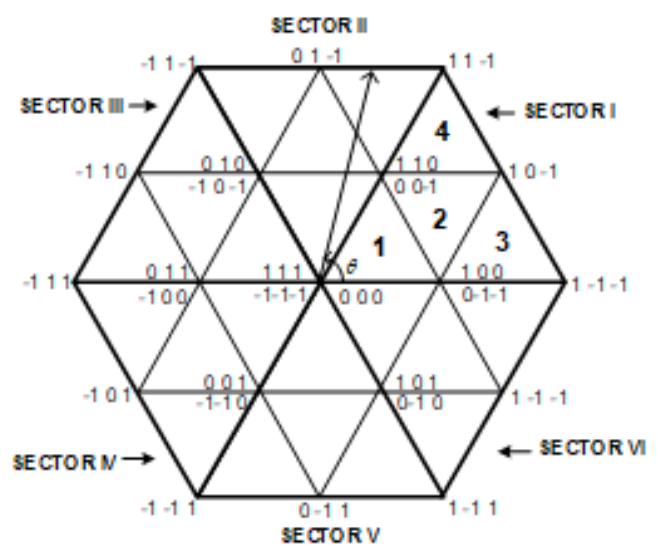

Fig. 3: Switching States of Three-level NPC Inverter Topology

one of the most popular modulation techniques in which a sinusoidal modulating (control) signal is compared with the triangular carrier signal as shown in Fig. 4. It works in a fashion when a modulating signal is higher than the carrier signal, the switch is ON otherwise OFF. For a positive half cycle of the modulating signal switches S1 and S3 are turned $\mathrm{ON}$ once in each control period and reverse is true for switches S2 and S4 in negative cycle. Besides, During positive cycle S2 and S4 remain invariant and keep themselves at 1 and 0 respectively, while the state of $S 1$ and $\mathrm{S} 3$ remain unchanged for negative cycle. 
IV. Neutral-Point Voltage Balance Control THROUGH ZERO-SEQUENCE VOLTAGE INJECTION

Switching states for the three-level NPC are expressed as:

$$
\begin{gathered}
V_{s s}=\left[S_{a}, S_{b}, S_{c}\right]^{T} \\
S_{x} \in[-1,0,1], x \in[a, b, c]
\end{gathered}
$$
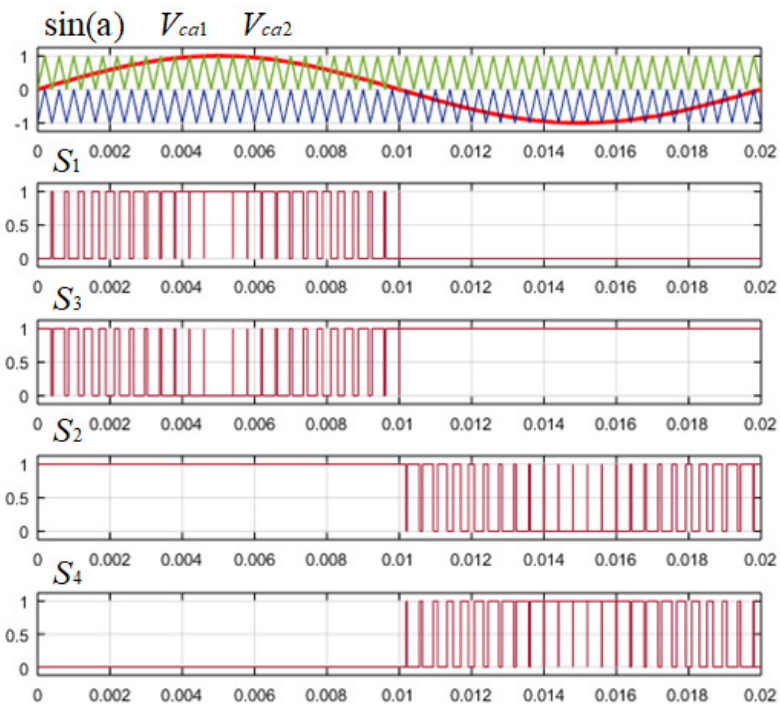

Fig. 4: Multi-carrier PWM Strategy for three-level NPC inverter

The load current of one phase which is clamped to neutral point flows out of the neutral point through the respective clamped diodes. This neutral current can be written as

$$
\begin{gathered}
i_{\text {neu }}=\left(1-\left|S_{a}\right|\right) i_{a}+\left(1-\left|S_{b}\right|\right) i_{b}+\left(1-\left|S_{c}\right|\right) i_{c} \\
\left.i_{\text {neu }}=-\left|S_{a}\right| i_{a}-\left|S_{b}\right|\right) i_{b}-\left|S_{c}\right| i_{c}
\end{gathered}
$$

The zero sequence voltages are injected into reference voltages given by

$$
\left\{\begin{array}{c}
U_{a}=e_{a}+U_{0} \\
U_{b}=e_{b}+U_{0} \\
U_{c}=e_{c}+U_{0}
\end{array}\right.
$$

The sign function can be defined as

$$
\operatorname{sgn}(v)=\left\{\begin{array}{l}
1 \quad v \geq 0 \\
-1 \quad v<0
\end{array}\right.
$$

Hence, average neutral current can be obtained by insert- ing (3) and (4) into (2)

$$
\begin{aligned}
& i_{\text {neu }}=-\left[\operatorname{sgn}\left(e_{a}\right) \cdot e_{a} \cdot i_{a}+\operatorname{sgn}\left(e_{b}\right) \cdot e_{b} \cdot i_{b}+\operatorname{sgn}\left(e_{c}\right) \cdot e_{c} \cdot i_{c}\right] \\
& -U_{0}\left[\operatorname{sgn}\left(e_{a}\right) \cdot i_{a}+\operatorname{sgn}\left(e_{b}\right) \cdot i_{b}+\operatorname{sgn}\left(e_{c}\right) \cdot i_{c}\right]
\end{aligned}
$$

In steady state, the average neutral current should be controlled to zero in order to implement the NP balancing control. The zero-sequence voltage can be controlled as

$$
U_{0}=-\frac{\operatorname{sgn}\left(e_{a}\right) \cdot e_{a} \cdot i_{a}+\operatorname{sgn}\left(e_{b}\right) \cdot e_{b} \cdot i_{b}+\operatorname{sgn}\left(e_{c}\right) \cdot e_{c} \cdot i_{c}}{\operatorname{sgn}\left(e_{a}\right) \cdot i_{a}+\operatorname{sgn}\left(e_{b}\right) \cdot i_{b}+\operatorname{sgn}\left(e_{c}\right) \cdot i_{c}}
$$

The neutral point voltage can be regulated by properly adjusting the neutral current. For simplification, the positive sequence modulating signal is divided into six different parts as shown in Fig. 5 .

It is clear from the Fig. 5 that only one of the phases has the maximum amplitude during each interval. For instance, Phase A has maximum amplitude during $\left[-30^{\circ}, 30^{\circ}\right]$, whereas phases $\mathrm{B}$ and $\mathrm{C}$ has the peak amplitudes during the intervals $\left[-90^{\circ}, 150^{\circ}\right]$ and $\left[-210^{\circ}, 270^{\circ}\right]$ respectively. It means there is specific phase current in each interval which influences the neutral point voltage. Each interval further consists of set of small vectors which can be controlled in order to maintain the neutral point potential, as given in Table 2.

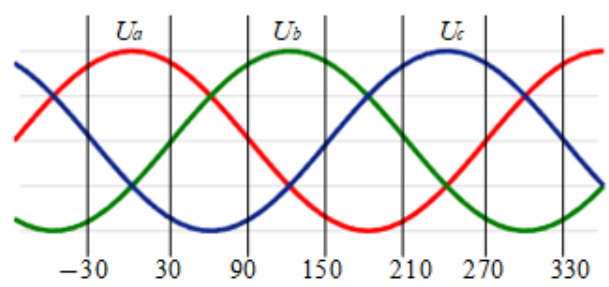

Fig. 5: Six Sectors of Modulation Voltage

Based on multi-carrier modulation method, the appropriate zero-sequence component can be estimated using current direction and difference of the two dc link capacitors. Injection of zero-sequence voltage into modulating voltage actually change the dwell time of the redundant small vectors and NP balance control is achieved. Adding positive zero sequence voltage increases the action time of the small vector type $\mathrm{P}$ whereas, the action time of the small vector type $\mathrm{N}$ rises by adding negative zero sequence voltage. For instance, during the interval $\left[-30^{\circ} \leq \theta \leq 30^{\circ}\right]$ if neutral point voltage is greater than the zero and its current direction is towards the neutral point, then by injecting positive sequence voltage the neutral point voltage can be reduced. On the other hand, if neutral point potential is less than zero and current direction 
TABLE I: Small Vector and corresponding current in each sector

\begin{tabular}{|c|c|c|c|c|}
\hline Sectors & P-Type Small Vector & $i_{\text {neu }}$ & N-type small vectors & $i_{\text {neu }}$ \\
\hline$-30^{\circ} \leq \theta \leq 30^{\circ}$ & {$\left[\begin{array}{lll}1 & 0 & 0\end{array}\right]$} & $-i_{a}$ & {$\left[\begin{array}{lll}0 & -1 & -1\end{array}\right]$} & $i_{a}$ \\
\hline $30^{\circ} \leq \theta \leq 90^{\circ}$ & {$\left[\begin{array}{lll}1 & 1 & 0\end{array}\right]$} & $i_{c}$ & {$\left[\begin{array}{lll}0 & 0 & -1\end{array}\right]$} & $-i_{c}$ \\
\hline $90^{\circ} \leq \theta \leq 150^{\circ}$ & {$\left[\begin{array}{lll}0 & 1 & 0\end{array}\right]$} & $-i_{b}$ & {$\left[\begin{array}{lll}-1 & 0 & -1\end{array}\right]$} & $i_{b}$ \\
\hline $150^{\circ} \leq \theta \leq 210^{\circ}$ & {$\left[\begin{array}{lll}0 & 1 & 1\end{array}\right]$} & $i_{a}$ & {$\left[\begin{array}{lll}-1 & 0 & 0\end{array}\right]$} & $-i_{a}$ \\
\hline $210^{\circ} \leq \theta \leq 270^{\circ}$ & {$\left[\begin{array}{lll}0 & 0 & 1\end{array}\right]$} & $-i_{c}$ & {$\left[\begin{array}{lll}-1 & -1 & 0\end{array}\right]$} & $i_{c}$ \\
\hline $270^{\circ} \leq \theta \leq 330^{\circ}$ & {$\left[\begin{array}{lll}1 & 0 & 1\end{array}\right]$} & $-i_{c}$ & {$\left[\begin{array}{lll}0 & -1 & 0\end{array}\right]$} & $i_{c}$ \\
\hline
\end{tabular}

is into the neutral point, then by adding zero sequence voltage the neutral point voltage can be increased. However, the injection of zero-sequence voltage should follow the constraint given,

$$
\left|e_{a}+U_{0}\right|<M
$$

The injection method of zero sequence voltage consists of following steps,

- Conversion of three phase modulation waveform from $a b c$ to $\alpha \beta$ plane.

- Find the angle of rotation $\theta$ through $\mathrm{U}_{\alpha}, \mathrm{U}_{\beta}$

- Finding out the sector using $\theta$

- Add the zero-sequence voltage based on the neutral point voltage and phase current direction.

- Finally, three-phase modulating signal is sent to modulation block to generate the PWM signal for IGBTs

It is observed that the balancing techniques for neutral point voltage use some sort of zero sequence voltage manipulation in case of carrier based PWM. Whereas, all NP balancing schemes in SVM are also based on the same concept of manipulating redundant small vector switching levels. Since the difference between the phase voltage of the positive and negative small vector in the same pair is zero sequence voltage, the small vector manipulation is the zero-sequence voltage manipulation. This appears to be the equivalence between NP balance control systems based on carrier and SVM based PWM schemes.

\section{Simulation Results}

The simulations of the Shunt APF connected to a threephase rectifier as non-linear load are performed in MATLAB/SIMULINK environment. The midpoint voltage control is based on a zero-sequence voltage injection using the carrier based PWM modulation scheme is shown in the Fig. 6. It can be seen that the maximum midpoint fluctuation voltage is $5 \mathrm{~V}$, which is reasonable. As the value of the dc side capacitor may not have exactly the same in the actual systems. In order to realize the efficacy of the control of the midpoint potential in extreme scenario, two capacitance values are set at $4000 \mathrm{~F}$ and $6000 \mathrm{~F}$, with initial voltages set at $412 \mathrm{~V}$ and $338 \mathrm{~V}$ respectively while keeping the remaining parameters unchanged. Under these circumstances, the simulation is given in Fig. 7, showing that both capacitors maintain the equal potential in just 0.08 s.
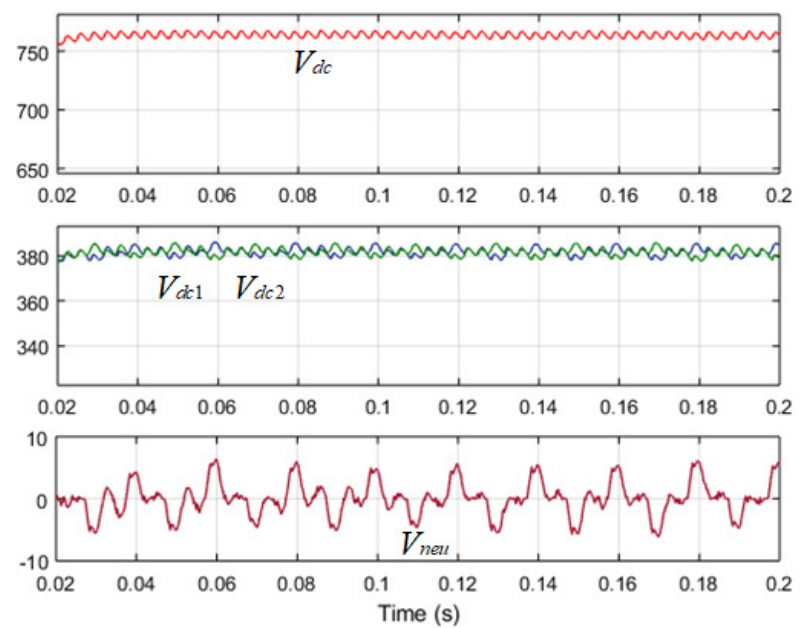

Fig. 6: Midpoint voltage control waveform with same capacitors value
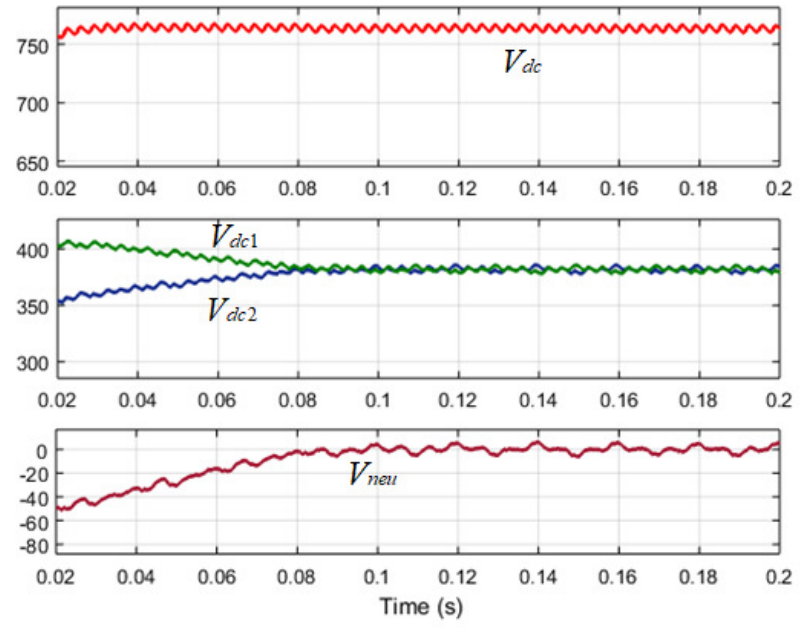

Fig. 7: Midpoint voltage control waveform with different capacitors value 
Fig. 8. shows DC-side voltage and the midpoint response during load changes suddenly, which shows the DC-side voltage and the midpoint voltage fluctuations also increase due to the sudden increase in the APF output current. This results in a sudden increase in the neutral current, nevertheless, the dc-side and midpoint potential fluctuations are still less than 5 (RMS). The results of the three-level APF compensation are provided in Fig. 9, which represent the load current, filter and grid currents. Approximately $90 \%$ harmonic reduction occurs after compensation can be clearly seen.
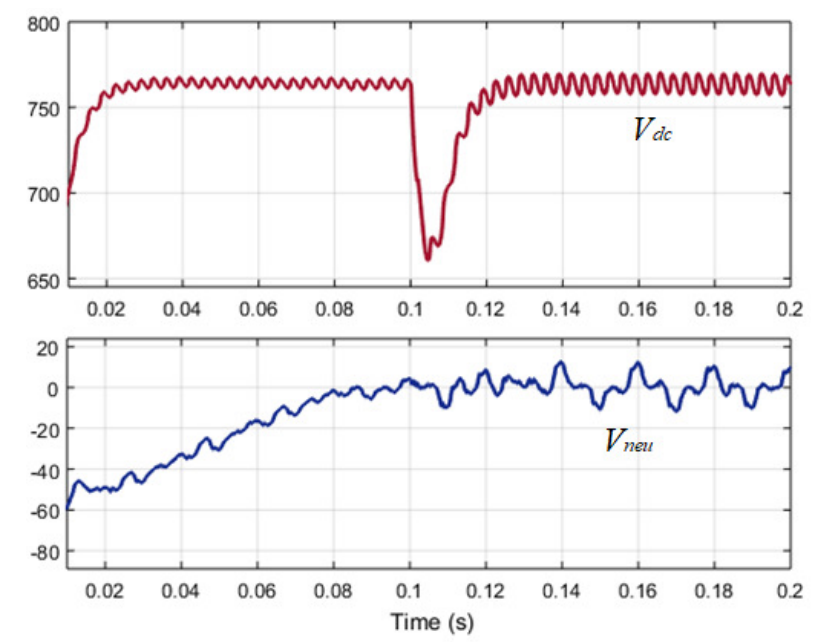

Fig. 8: DC side voltage and neutral point voltage during load change
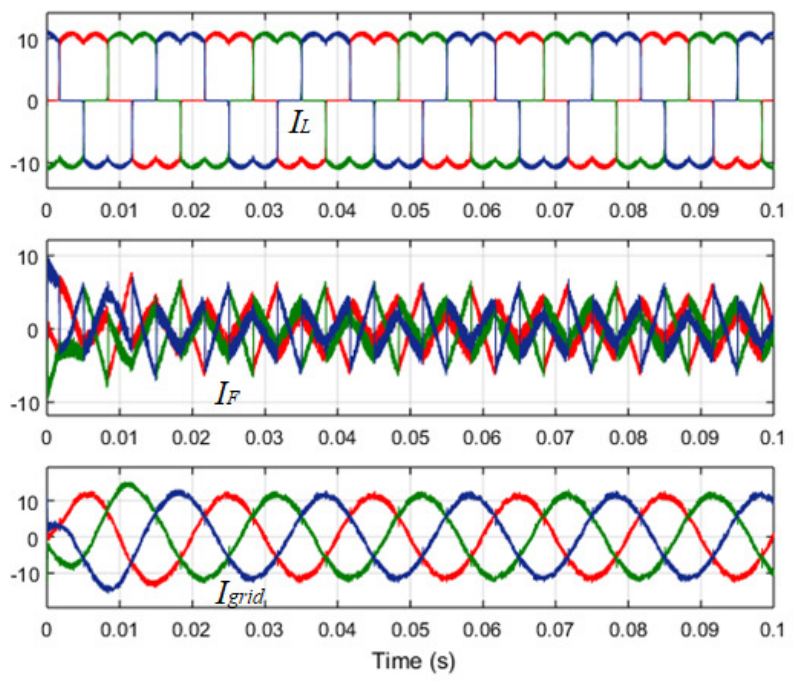

Fig. 9: Compensation effect of three-level APF

\section{CONCLUSIONS}

This paper demonstrated the neutral point voltage deviation control in three-level NPC inverter based shunt APF.
By using the voltage difference of two DC-link capacitor and the direction of neutral current, a zero sequence component is estimated and added to modulating signal before sending it to carrier-based PWM. This zero-sequence component manipulates the action time of the small vector and hence regulates the neutral point voltage near to zero. Consequently, voltage across each capacitor maintain exactly half of the total DC-link voltage. The simulation results confirms the effectiveness of the presented method in shunt APF compensating the harmonics to mimimum with overall THD reduced to less than $5 \%$.

\section{REFERENCES}

[1] Kashif, Muhammad, M. J. Hossain, Yuba Raj Kafle, and Md Shamiur Rahman. "A comparative study of two current-control techniques applied to a three-phase three-level active power filter." In 2017 IEEE International Telecommunications Energy Conference (INTELEC), pp. 357-362. IEEE, 2017.

[2] Tareen, Wajahat Ullah Khan, and Saad Mekhielf. "Three-phase transformerless shunt active power filter with reduced switch count for harmonic compensation in grid-connected applications." IEEE Transactions on Power Electronics 33, no. 6 (2018): 4868-4881.

[3] Gautam, Samir, Pei Yunqing, Yubaraj Kafle, Muhammad Kashif, and Saad Ul Hasan. "Evaluation of fundamental dq synchronous reference frame harmonic detection method for single phase shunt active power filter.' International Journal of Power Electronics and Drive Systems 4, no. 1 (2014): 112.

[4] Kashif, Muhammad, M. J. Hossain, Fang Zhuo, Shuhuai Shi, and John Long Soon. "An advanced harmonic extraction technique applied to a three-phase three-level active power filter." In 2017 IEEE 3rd International Future Energy Electronics Conference and ECCE Asia (IFEEC 2017-ECCE Asia), pp. 364-369. IEEE, 2017.

[5] Kashif, Muhammad, M. J. Hossain, Fang Zhuo, and Samir Gautam. "Design and implementation of a three-level active power filter for harmonic and reactive power compensation." Electric Power Systems Research 165 (2018): 144-156

[6] Akagi, Hirofumi. "Multilevel converters: Fundamental circuits and systems." Proceedings of the IEEE 105, no. 11 (2017): 2048-2065

[7] Kashif, Muhammad, Zhuo Fang, Samir Gautam, Yu Li, and Ali Syed. "A study of three-level neutral point clamped inverter topology." Indonesian Journal of Electrical Engineering and Computer Science 12, no. 8 (2014): 5999-6008.

[8] Pou, Josep, Dushan Boroyevich, and Rafael Pindado. "Effects of imbalances and nonlinear loads on the voltage balance of a neutralpoint-clamped inverter.” IEEE Transactions on power electronics 20.1 (2005): 123-131.

[9] Song, Qiang, et al. "A neutral-point potential balancing algorithm for three-level NPC inverters using analytically injected zero-sequence voltage." Eighteenth Annual IEEE Applied Power Electronics Conference and Exposition, 2003. APEC'03.. Vol. 1. IEEE, 2003.

[10] Jinghua, Zhou, et al. "A neutral-point potential control method for three-level inverters by injecting zero-sequence voltage." The 2nd International Symposium on Power Electronics for Distributed Generation Systems. IEEE, 2010.

[11] Hoon, Yap, et al. "Neutral-point voltage deviation control for threelevel inverter-based shunt active power filter with fuzzy-based dwell time allocation." IET Power Electronics 10.4 (2016): 429-441. 